\title{
Analysis and Design of a Novel Metamaterial-Based Microstrip Antenna
}

\author{
Shiquan Zhang \\ Department of Science \\ Engineering University of PAP \\ Xi'an, China
}

\begin{abstract}
Two-layer lattice-type metamaterials unit is loaded above the coaxial feed microstrip antenna in order to gather electromagnetic wave by using the zero refraction index effect. The $\pi$-type metamaterials unit is printed on the antenna substrate, and the band gap effect is used to suppress the backward wave of the antenna. Experiment and analysis are made on the two optimization methods. Finally two methods are combined in designing a microstrip antenna with high directivity and high gain. Comparing the results of the results of simulation experiment with those of the real test, the correctness of the theory and the design idea is verified. As combined with the traditional antenna, the gain of the new microstrip antenna has achieved by $8.94 \mathrm{~dB}$, an increase $51.27 \%$ and the directivity iis increased by $48.89 \%$, thus achieving the goal of the designed experiment.
\end{abstract}

Keywords - metamaterials; microstrip antenna; high directivity and gain

\section{INTRODUCTION}

In recent years, microstrip antenna has been extensively applied in different fields of electronic engineering because of its advantages such as small size and easiness to integration. However, its disadvantages of low directivity and high loss restrain its use. With constantly higher requirements for the antenna performance by various devices, the design of highgain and high-directivity microstrip antenna has become the focus of studies. Traditional optimization methods cannot go beyond the bottleneck of interactive restraint between physical size, bandwidth and gain, etc. Hence, since metamaterials were produced in 2001, the use of metamaterials to improve the antenna performance had attracted extensive attentions ${ }^{[1-4]}$. A covering layer is loaded above the antenna with the zero refraction index features of metamaterials or band gap substrate is loaded above the antenna, which all can improve the gain and directivity of antenna to some extent. Yet, there are few studies on the analysis of antenna performance based on the combination of two methods above. Moreover, discussions about the influence of distance of the antenna covering layer fall short. In this paper, a kind of microstrip antenna with both covering layer and substrate made out of metamaterials is designed; and detailed analysis is conducted on the influential parameters of the antenna covering layers. The result shows that the gain and directivity of antenna with both covering layer and substrate made out of metamaterials have been obviously enhanced compared with traditional microstrip antenna.

\section{ANTENNA DESIGN AND ANALYSIS}

We determine concrete requirements first, then design unit structure and adjust parameters to meet with corresponding demands. Through calculation and the experiment simulation adjustment, FR4 with a dielectric constant $\varepsilon_{p}=4.4$ and thickness $\mathrm{h}=1.6 \mathrm{~mm}$ was selected to be the substrate of the antenna and a kind of coaxial feed microstrip antenna with a central working frequency of was designed. The substrate size is $48 \mathrm{~mm} \times 40 \mathrm{~mm}$. The size of the antenna radiation patch is $16 \mathrm{~mm} \times 10.8 \mathrm{~mm}$. The distance between coaxial feeding point and the center of the patch is $L=2.7 \mathrm{~mm}$. ${ }^{50 \Omega}$ coaxial feed model is used for the antenna.

\section{A. Metamaterials Unit Design}

Fig. 1 and Fig. 2 show structures of the lattice-type and $\pi-$ type metamaterials unit designed in this paper. Table 1 and table 2 show their respective size.

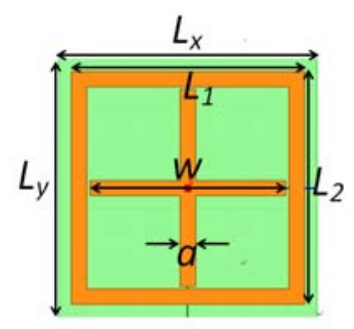

Fig. 1 Lattice-type metamaterials unit structure

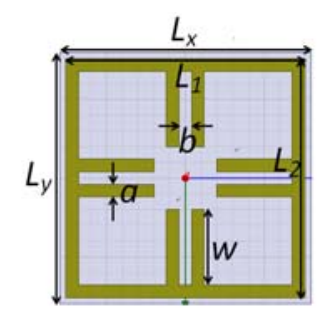

Fig. $2 \pi$-type metamaterials unit structure 
TABLE I SIZE PARAMETERS OF THE LATTICE-TYPE UNIT

\begin{tabular}{|c|c|c|c|}
\hline$L_{x}$ & $8 \mathrm{~mm}$ & $L_{y}$ & $8 \mathrm{~mm}$ \\
\hline$L_{1}$ & $7.5 \mathrm{~mm}$ & $L_{2}$ & $7.5 \mathrm{~mm}$ \\
\hline$w$ & $6 \mathrm{~mm}$ & $a$ & $0.5 \mathrm{~mm}$ \\
\hline
\end{tabular}

TABLE II SIZE PARAMETERS OF THE П-TYPE UNIT

\begin{tabular}{|c|c|c|c|}
\hline$L_{x}$ & $8 \mathrm{~mm}$ & $L_{1}$ & $7.5 \mathrm{~mm}$ \\
\hline$L_{y}$ & $8 \mathrm{~mm}$ & $L_{2}$ & $7.5 \mathrm{~mm}$ \\
\hline$w$ & $2.8 \mathrm{~mm}$ & $a$ & $0.4 \mathrm{~mm}$ \\
\hline$b$ & $0.4 \mathrm{~mm}$ & & \\
\hline$b$ & $0.4 \mathrm{~mm}$ & & \\
\hline
\end{tabular}

Six lattice-type metamaterials units were lined in the way of $2 * 3$ and plated on the FR4 substrate with a thickness of $1.6 \mathrm{~mm}$ as the covering layer of the antenna. HFSS software was used for the simulation analysis of the metamaterials covering layer. See Fig. 3 for S parameter. Smith method was applied to extract data obtained from Fig. $3^{[5-6]}$ to obtain the effective refraction index of the covering layer in Fig. 4. It can be seen from Fig. 4 that the refraction rate of the covering layer at the point of $6 \mathrm{GHz}$ is close to zero, which is in line with the design need.

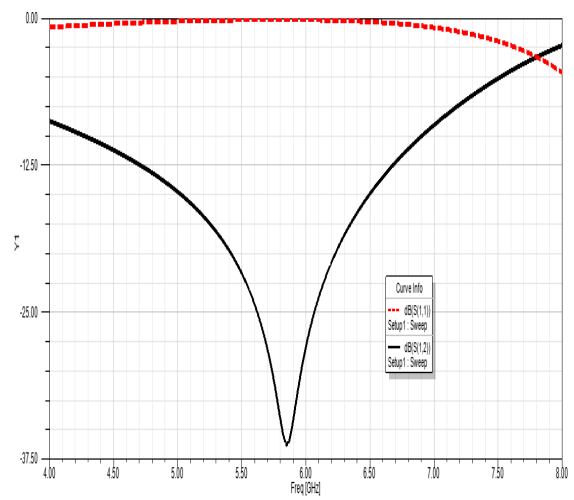

Fig. $3 \mathrm{~S}$ parameter of the lattice-type unit

$\pi$-type metamaterial is pated around the antenna substrate and the $\mathrm{S}$ parameter after simulation is shown in Fig. 5. It can be noted that the peak value of the transmission band gap of the substrate is $-24.5 \mathrm{~dB}$ at the point of $6 \mathrm{GHz}$. It can be used to suppress the backward wave of the antenna and it conforms to the requirements of the designed antenna.

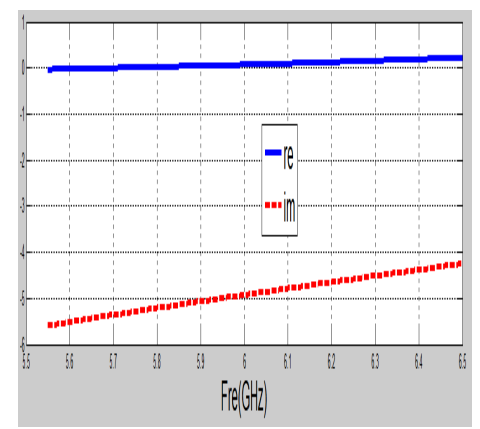

Fig. 4 Refraction rate of the lattice-type unit

\section{B. Metamaterial-based Microstrip Antenna}

$\pi$-type metamaterial unit is periodically loaded on the microstrip antenna substrate around the patch to obtain the antenna indicated in Fig. 6. Then the two layer lattice-type metamaterials covering layer is supported by foam and placed right above the microstrip antenna. The distance between the lower layer and the antenna is sjuli $=25 \mathrm{~mm}$, that is, half wavelength. The distance between the two covering layers is $\mathrm{B}=5 \mathrm{~mm}$. See Fig. 7 for the comprehensively designed antenna structure.

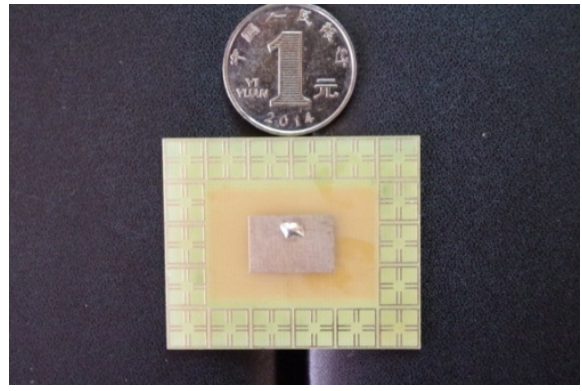

Fig. 6 Antenna with metamaterial substrate

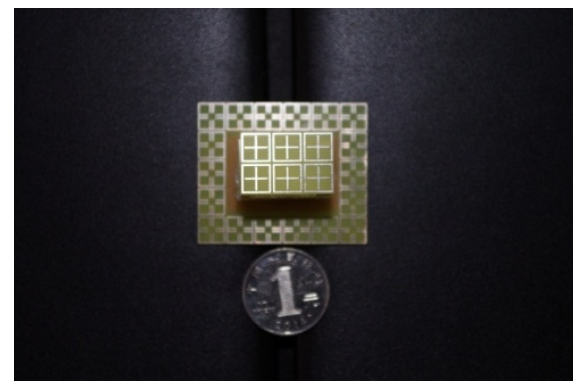

Fig. 7 Comprehensively designed physical antenna

1) Return Loss: The return loss of the original antenna, antenna with metamaterials substrate and comprehensively designed antenna obtained from the simulation and material experiments can be seen in Fig. 8. As indicated in the figure, compared with the original antenna, after metamaterial is loaded, the return loss of the antenna deteriorates because of certain loss of the metamterial itself so small deviation happens in the central frequency.

2) Analysis of the Gain :I t can be seen from Fig. 9 that the gain of the antenna increase significantly when it's about $\pm 100^{\circ}$, which means the metamaterials substrate can well restrain the backward wave. The designed covering layer area cannot completely cover the antenna surface so sidelobe appears in the directivity figure of the comprehensively designed antenna. The antenna gain is increased by $51.27 \%$.

3) Directivity: Fig. 10 shows the directivity of the antenna. It can be noted from the figure that the directivity of the antenna is $10.69 \mathrm{~dB}$, an increase of $3.51 \mathrm{~dB}$ compared with the original antenna and by $48.89 \%$; an increase of $3.11 \mathrm{~dB}$ 
compared with the metamaterials substrate antenna and by $41.03 \%$.

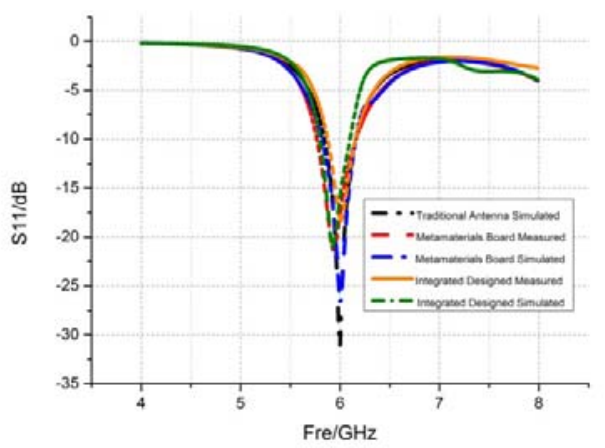

Fig. 8 Return loss of the antenna

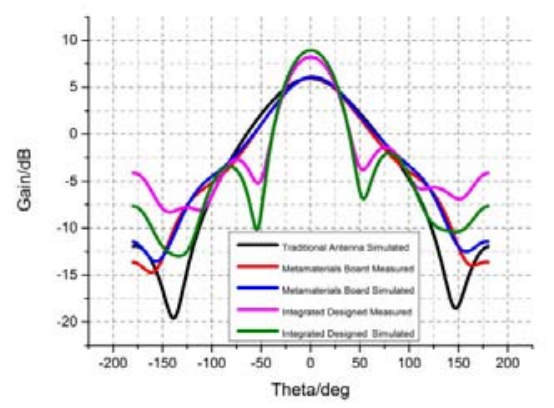

Fig. 9 Gain

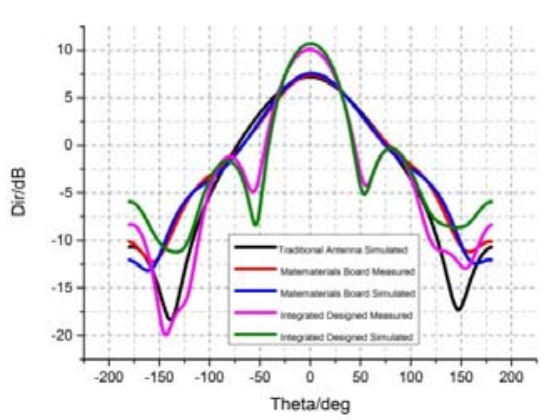

Fig. 10 Directivity of the designed antenna

\section{Conclusions}

Two different metamateirals units are loaded on the common coaxial feed microstrip antenna.The zero refraction index feature of metamaterials are used to work out the antenna covering layer to restrain the surface wave of the antenna substrate through directed radiation of electromagnetic wave and the band gap feature and to reduce the back wave energy loss of the antenna for the purpose of enhancing the antenna directivity and gain. The experiment result shows that the gain of the comprehensively designed antenna is $8.94 \mathrm{~dB}$, an increase of $3.03 \mathrm{~dB}$ compared with original antenna and by $51.27 \%$. Besides, the half-power beam width $(-3 \mathrm{~dB})$ of $\mathrm{E}$ surface and $H$ surface is respectively $47.87^{\circ}$ and $51.42^{\circ}$, a respective decrease of $40.42^{\circ}$ and $25.76^{\circ}$ compared with original antenna. The forward direction radiation of the antenna increases and the backward wave radiation are effectively restrained as well. The directivity of the antenna is $10.69 \mathrm{~dB}$, an increase of $3.51 \mathrm{~dB}$ compared with the original antenna and by $48.89 \%$, with its performance being greatly enhanced.

\section{ACKNOWLEDGMENT}

This work was supported by the National Natural Science Foundation of China (61072034), the National Natural Science Foundation of China (51302318), the provincial Natural Science Foundation of Shaanxi (2011JQ6013) and the Basic Research Project of Engineering University of CAPF (WJY201404, WJY-201405, WJY-201406).

\section{REFERENCES}

[1] R. A. Shelby, D. R. Smith. "Experimental verification of a negative index of refraction". Science.2001.292 (6): pp.77-79.

[2] YANG R, XIE Y, WANG P, et al. "Characteristics of millimeter wave microstrip antenna with left-handed materials substrates". Appl. Phys. Lett, 2006, 89: 064108(1-3).

[3] MOSAlLAEI H, SARABANDI K. "Antenna miniaturization and bandwidth enhancement using a reactive impedance substrate". IEEE Trans Antennas Propag., 2004,52(9): pp. 2403-2414.

[4] LIU Y H, ZHAO X P. "Inveatigation of anisotropic negative permeability medium cover for patch antenna". IET Microwaves, Antennas and Propagation, 2008. 7(2): pp.737-744.

[5] D.R. Smith, J.B. Pendry, and M.C.K. Wiltshire. "Metamaterials andnegative refractive index". Science 305 (2004): pp. 788-792.

[6] D. R. Smith and S. Schultz. "Determination of effective permittivity and permeability of metamaterials from reflection and transmission coefficients". Physical Review B, 65, 195104 (2002). 